\title{
Fühl dich wohl in deiner Haut!
}

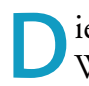
ie Autorinnen Bock, Ehlers und Worm bringen in einem nicht $\mathrm{zu}$ umfangreichen Büchlein Schulkindern und deren Eltern in kurzweiliger Form ausgewählte Aspekte des täglichen Umgangs mit der Neurodermitis nahe. Die Einführung der „Murmis“ zur kindlichangepassten Pathogenese-Erläuterung ist ungewöhnlich, könnte jedoch für manchen Patienten eine einfache Erklärung für die ansonsten nur schwer nachvollziehbaren Neurodermitis-Schübe darstellen. Der durchweg ansprechend geschriebene Text erläutert anschaulich am Beispiel der kleinen Anna die in der ärztlichen Praxis durchgeführten, für die Diagnostik des atopischen Ekzems wesentlichen Prozeduren: Blutabnahme für eine In-vitro-IgE-Diagnostik, Pricktestung und vor allem die Nahrungsmittel-Provokation. Auch auf externe Provokationsfaktoren wie Aero- und Nahrungsmittel-Allergene, Diät-Tagebuch und die regelmäßige äußerliche Therapie mit indifferenten Externa gehen die Autorinnen ein.

Die großflächige und durchweg ansprechende Bebilderung macht das Buch leicht lesbar und anschaulich. Text und Abbildungen des Hauptteils werden durch einen kleiner gedruckten, wissenschaftlich gehaltenen Anhang für die Eltern sinnvoll ergänzt. Einen Ersatz für die bekannten ausführlicheren und vor allem für Eltern und wissenschaftlich interessierte Patienten geschriebenen Bücher und Lexika zum atopischen Ekzem kann und will dies Büchlein nicht darstellen. Die durchweg rational strukturierte und auf Evidenz-basierten Lehrinhalten fundierte Broschüre kann aber sowohl für die kleinen Patienten und ihre Eltern hilfreich sein als auch für die Arztpraxis als Lektüre im Wartezimmer gute Dienste leisten. PD Dr. A. Wollenberg, München

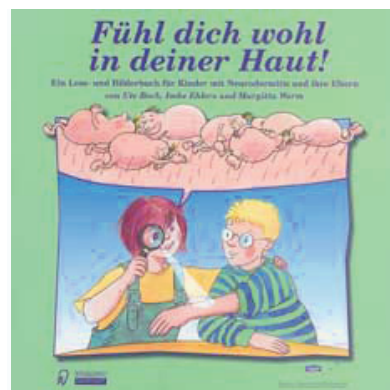

Bock U, Ehlers I, Worm M.

Fühl dich wohl in deiner Haut. Ein Lese- und Bilderbuch für Kinder mit Neurodermitis und ihre Eltern.

Darmstadt: Steinkopff 1999; 60 Seiten, 17,50 DM

ISBN 3-798511-57-8

\section{Für Pollen-Detektive auf der Wunschliste}

$\mathrm{D}$ ieser nun endlich vorliegende, handliche, Pollen-diagnostische Atlas bedeutet einen weiteren Fortschritt in der Qualitätssicherung von Pollen-Analysen in Deutschland. Er ist aus der Praxis für die Praxis verfasst und wendet sich an alle, die für den PollenInformationsdienst (PID) Pollen-Bestimmungen vornehmen, an Allergologen, interessierte Ärzte und von der Pollinose Betroffene. Ein gelungener Überblick mit vielen Hinweisen: Das alphabetische Register umfasst die Pollen-Arten nach ihren deutschen Pflanzennamen und wird ergänzt durch die lateinischen und englischen Namen, die verwandtschaftliche Zugehörigkeit einzelner Pollen-Arten zu bestimmten Pflanzen-Familien, ihre allergologische Bedeutung. Folgende Gesichtspunkte wurden berücksichtigt: Häufigste in Mitteleuropa vorkommende aerogene

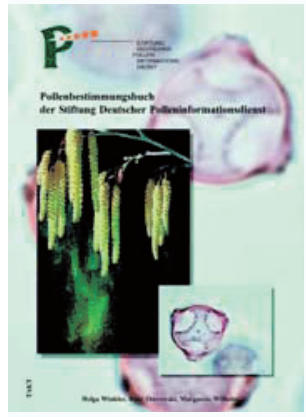

Winkler $\mathrm{H}$, Ostrowski R, Wilhelm $M$.

Bergmann K-Ch, Hrsg.

Pollenbestimmungsbuch der Stiftung Deutscher PollenInformationsdienst. Paderborn: TAKT-Verlag, 2001; 80 Seiten, 133 Abbildungen, 34 Euro

ISBN 3-931732-09-6

Pollenarten, Beschreibung der zugehörigen Pflanzen und Blüten mit Abbildungen, lichtmikroskopische Abbildungen der Pollen, entsprechend dem Erscheinungsbild bei der täglichen Pollendiagnose, durchschnittliche Größenangaben und morphologische Charakteristika.

Prof. Dr. E. Fuchs, Detmold 